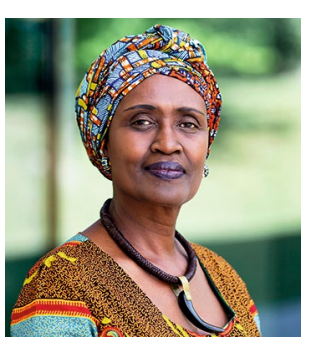

\title{
HIV or COVID-19, inequity is deadly
}

\author{
Breaking pharmaceutical monopolies helped to address the HIV crisis. The same could be \\ done to end the COVID-19 pandemic, but we must act decisively, writes Winnie Byanyima.
}

Credit: UNAIDS

$\Lambda$ $s$ we embark on a new year of the battle between humanity and COVID-19, the virus is still winning. Leaders of high-income nations have made huge and ultimately inexcusable errors of judgement. They have failed to consider vaccines and COVID-19 technologies as global public goods.

We are witnessing the same deadly mistakes made in the mid-1990s, when treatment for HIV became available. Pharmaceutical companies set the extortionate price tag of US $\$ 10,000$ per person per year, rendering this life-saving treatment out of reach for the millions of people living with HIV in the Global South. Between 1997 and 2006, UNAIDS estimates that 12 million African individuals died because the medicines were priced out of their reach by pharmaceutical monopolies and the greed of profit versus public good.

It was only when a movement of people living with HIV, healthcare advocates, religious leaders and many other partners mobilized to generate global political pressure that companies in developing countries (Brazil, India and Thailand) were able to manufacture the medicines by making use of the flexibilities within the World Trade Organization's (WTO) Trade-Related Aspects of Intellectual Property Rights Agreement.

This important tool allowed for competition within the pharmaceutical market, which led to dramatic drops in the price of HIV medicines to less than US \$75 in some countries today, giving millions access to life-saving treatment. But this took years to achieve, and many countries still cannot access the generic market owing to trade barriers. We cannot let that happen with COVID-19, and this time we must act much faster.

However, the world is currently failing to heed the lessons of the injustice that took place with the AIDS pandemic, and leaders are still putting narrow and ultimately self-defeating nationalism ahead of what is needed. They are continuing to defend the profits and monopolies of their pharmaceutical corporations, instead of sharing the successful vaccines and the technologies that would scale up their production worldwide.

It has been a year since the first Pfizer-BioNTech vaccine was given, and yet Africa has still only received enough doses to fully vaccinate around $10 \%$ of its population. This is in stark contrast to high-income countries. In the European Union, for example, around $70 \%$ of people have received at least two doses, with many countries now administering third booster jabs to keep their populations safe.

High-income nations have behaved appallingly, hording billions of doses of vaccines while doctors in countries such as my own, Uganda, have faced COVID-19 unprotected and unvaccinated. Even when high-income countries do donate some of their excess doses, it is too little, too late often delivered close to their expiry dates, and virtually unusable. Donations and charity, though welcome, will never be enough.

At least five million people have already died of COVID-19, while world leaders have allowed companies such as Pfizer and Moderna to make as much as US $\$ 1,000$ profit a second, hiding behind their monopolies to artificially restrict the supply of these vaccines and making them the most lucrative medicine ever developed creating new vaccine billionaires instead of vaccinating billions.

Putting profits first has also created an optimal breeding ground for new variants, such as Omicron. One thing we know for sure is that the impact of Omicron will be felt by the poorest worldwide. The poorest people and low-income nations will be least able to take the actions needed to combat it.

We know that already scarce vaccines will become hugely scarcer. Being fully vaccinated will soon mean having multiple doses, leading to higher-income nations buying up all the vaccines for boosters. We may discover that some existing vaccines are not able to face the challenge of new variants, and, as new vaccines are developed, existing supply capacity will be diverted to this aim.

There are steps we can, and must, take to help to fix this. The proposed temporary intellectual property waiver covering all COVID-19 vaccines and technologies at the WTO remains a necessary precondition to defeat COVID-19. The waiver could curb pharmaceutical companies' global monopolies, while still allowing them to be financially compensated.

We also need to invest now in building vaccine-production capacity all over the world, especially for the very successful mRNA vaccines. There are more than 100 producers worldwide that could be making the Pfizer-BioNTech vaccine today. Know-how must be shared: these vaccines have been paid for by public money and they must be made a global public good.

Everyone has the right to health; we cannot afford to make the same mistakes we did in response to the emergence of HIV. We need greater global equity in healthcare and in access to health commodities and technologies, and we need it quickly.

It is madness to think that if we keep doing the same thing we can expect a different outcome. This year has to be the year that we finally make these incredible vaccines a global public good - the year that high-income nations do the right thing and that pharmaceutical companies share their successful vaccine recipes with producers all over the world.

Winnie Byanyima ${ }^{凶}$

UNAIDS, Geneva, Switzerland.

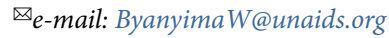

Published online: 31 January 2022

https://doi.org/10.1038/s41562-022-01307-9

Competing interests

The author declares no competing interests. 